\title{
Sigmoid volvulus: Comorbidity with sigmoid gangrene
}

\author{
Sabri Selcuk Atamanalp ${ }^{1}$, Esra Disci², \\ Refik Selim Atamanalp ${ }^{3}$
}

\begin{abstract}
Sigmoid volvulus (SV) is the wrapping of the sigmoid colon around its mesentery, and sigmoid gangrene is a catastrophic complication of SV. Although the diagnosis of SV is generally not difficult, unfortunately, most of the clinical, laboratory and radiological signs are not pathognomonic in demonstrating sigmoid gangrene. The treatment of gangrenous SV requires emergency surgery. Sigmoid gangrene worsens the prognosis of SV by doubling the mortality rate.
\end{abstract}

KEY WORDS: Sigmoid Colon, Volvulus, Sigmoid Gangrene, Endoscopy, Surgery.

doi: https://doi.org/10.12669/pjms.35.1.295

How to cite this:

Atamanalp SS, Disci E, Atamanalp RS. Sigmoid volvulus: Comorbidity with sigmoid gangrene. Pak J Med Sci. 2019;35(1):288-290. doi: https://doi.org/10.12669/pjms.35.1.295

This is an Open Access article distributed under the terms of the Creative Commons Attribution License (http://creativecommons.org/licenses/by/3.0), which permits unrestricted use, distribution, and reproduction in any medium, provided the original work is properly cited.

\section{INTRODUCTION}

Sigmoid gangrene is seen in $6.1-93.4 \%$ of cases with sigmoid volvulus (SV). ${ }^{1,2}$ Although some clinical and laboratory findings such as melanotic stool, fever, leucocytosis, abdominal guarding/rebound tenderness, hypotension/shock, somnolence and metabolic acidosis suggest the sigmoid gangrene, most of them generally fail in accurate diagnosis. ${ }^{3,4}$ Similarly, although some radiological studies including Doppler ultrasonography, angiography

1. Prof. Sabri Selcuk Atamanalp, MD.

2. Esra Disci, MD.

Assistant Professor

3. Refik Selim Atamanalp, MD.

Assistant Professor,

1-3: Department of General Surgery,

Faculty of Medicine,

Ataturk University,

Erzurum, Turkey.

Correspondence:

Prof. Sabri Selcuk Atamanalp, MD.

Department of General Surgery,

Faculty of Medicine,

Ataturk University, 25040,

Erzurum, Turkey.

E-mail: ssa@atauni.edu.tr

* Received for Publication:

* Accepted for Publication: or scintigraphy demonstrate the vascular occlusion, they are generally inadequate in determining sigmoid gangrene. ${ }^{5}$ When considerations are suitable, endoscopy identifies mucosal viability. ${ }^{6}$ Emergency surgery is needed to treat gangrenous SV, and primary anastomosis or stoma is used together with the resection of the gangrenous sigmoid colon. ${ }^{7,8}$

Despite SV being rare worldwide, ${ }^{3}$ it is relatively common in Eastern Anatolia, ${ }^{9}$ where we live. Our clinic has approximately 52 years of history and 1,008 cases of experience with SV, which is the largest single-centre SV series over the world. ${ }^{10} \mathrm{We}$ wanted to utilize the present data and experience to evaluate the comorbidity of SV with sigmoid gangrene.

\section{CLINICAL EXPERIENCE}

In our 1,008-case SV series, sigmoid gangrene was determined in a total of 284 patients $(28.2 \%)$. Diagnosis was made by digital rectal examination in 96 cases, by endoscopy (Fig.1) in 44, and by laparotomy (Fig.2) in the remaining. In our experiments, sigmoid gangrene is generally limited to the twisted segment or a few $\mathrm{cm}$. from the proximal and distal lines, but it rarely extends 


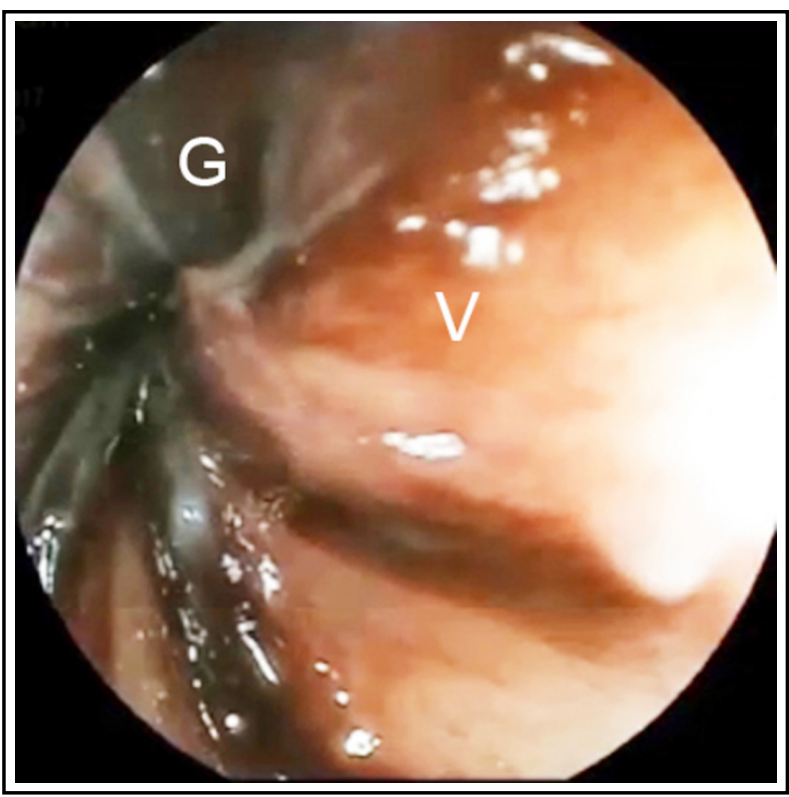

Fig.1: Endoscopic appearance of sigmoid gangrene in sigmoid volvulus.

(G: gangrenous mucosa, V: viable mucosa).

to the descending colon or rectum, only occurring in patients with very late admission, over-rotation or ileosigmoid knotting.

\section{DISCUSSION}

In SV, sigmoid gangrene is seen in $6.1-30.2 \%$ of all patients and in 10.7-93.4\% of surgically treated cases. ${ }^{1,2}$ Well-known factors affecting bowel gangrene development are the presence of major comorbidities (e.g., cardio-vascular diseases), shock (hypovolemic and/or toxic), delayed admission to the hospital, over-rotation (twisting degree $>360^{\circ}$ ), and ileosigmoid knotting. ${ }^{2,3,11,12}$

In SV, clinical presentation originally arises from the precursor pathology, acute closedloop intestinal obstruction. Additionally, the bloodstream is decreased or interrupted by the twisting of the mesentery. If the process continues for a few hours, the secondary pathology, bowel ischaemia and gangrene, and related clinical features are supervened. ${ }^{4,13}$ In addition to the initial hypovolemia, due to volume loss into the obstructive bowel lumen, necrosis facilitates bacterial translocation and the absorption of toxic products, resulting in toxaemia. ${ }^{2}$

The prominent clinical and laboratory indicators of sigmoid gangrene are melanotic stool, fever, leucocytosis, abdominal guarding/rebound tenderness, hypotension/shock, somnolence and

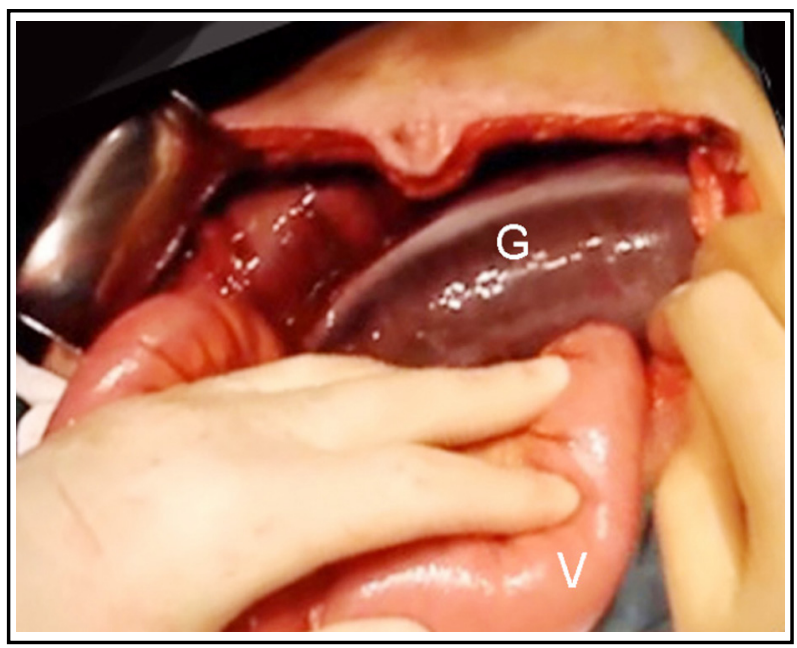

Fig.2: Operative appearance of sigmoid gangrene in sigmoid volvulus

(G: gangrenous sigmoid colon, V: viable small intestine)

metabolic acidosis. Except for the melanotic stool, these indicators are not pathognomonic., ${ }^{3,4}$ Doppler ultrasonography, angiography or scintigraphy may demonstrate the vascular occlusion, but this is not a mystery. ${ }^{5}$ If there is no contraindication, sigmoidoscopy or colonoscopy is a unique method to identify the mucosal viability. ${ }^{6}$ Endoscopic signs of bowel gangrene include devitalized brown-black mucosa and gangrenous blood-stained effluent., In surgery, bowel gangrene is easily diagnosed by means of a brown-black bowel segment. ${ }^{8}$

In SV, when bowel gangrene is determined or suspected in the preoperational period, emergency surgery is required without trying an endoscopy. If gangrene is diagnosed in an endoscopy, the procedure is terminated, and emergency surgery is planned. In operation, following the resection of the gangrenous segments, primary anastomosis is preferred in non-elderly and well-conditioned patients, while stoma may be life-saving in some elderly or bed-conditioned cases.,8,13,14 Bowel gangrene worsens the prognosis by increasing the mortality rate from $0-40 \%$ to $3.7-80 \% .2,9$

\section{CONCLUSION}

Sigmoid gangrene is a life-threating complication of SV. Even if the prognosis is generally poor, the observation of the basic diagnostic indicators and following the above mentioned basic therapeutic rules can relatively reduce the prognosis.

\section{Declaration of Interest: None.}

Grant Support E Financial Disclosure: None. 


\section{REFERENCES}

1. Vogel JD, Feingold DL, Stewart DB, Turner JS, Boutros M, Chun J, et al. Clinical practice guidelines for colon volvulus and acute colonic pseudo-obstruction. Dis Colon Rectum 2016;59(7):589-600. doi: 10.1097/DCR.0000000000000602.

2. AtamanalpSS, Kisaoglu A, Ozogul B. Factors affecting bowel gangrene development in patients with sigmoid volvulus. Ann Saudi Med. 2013;33(2):144-148. doi: 10.5144/02564947.2013.144.

3. Raveenthiran V, Madiba TE, Atamanalp SS, De U. Volvulus of the sigmoid colon. Colorectal Dis. 2010;12(7):e1-e17. doi: 10.1111/j.1463-1318.2010.02262.x.

4. Atamanalp SS. Sigmoid volvulus: diagnosis in 938 patients over 45.5 years. Tech Coloproctol. 2013;17(4):419-424. doi: 10.1007/s10151-012-0953-z.

5. Atamanalp SS, Atamanalp RS. What is done when endoscopic examination reveals borderline bowel ischemia in patients with sigmoid volvulus? Pak J Med Sci. 2017;33(3):761-763. doi: 10.12669/pjms.333.12265.

6. Atamanalp SS, Atamanalp RS. The role of sigmoidoscopy in the diagnosis and treatment of sigmoid volvulus. Pak J Med Sci. 2016;32(1):244-248. doi: 10.12669/pjms.321.8410.

7. Perrot L, Fohlen A, Alves A, Lubrano J. Management of the colonic volvulus in 2016. J Visc Surg. 2016;153(3):183-192. doi: 10.1016/j.jviscsurg.2016.03.006.

8. Atamanalp SS. Tretment of sigmoid volvulus: a single-center experience of 952 patients over 46.5 years. Tech Coloproctol 2013;17(5):561-569. doi: 10.1007/s10151-013-1019-6.

9. Atamanalp SS. A new classification, treatment algorithm and prognosis estimating system for ileosigmoid knotting. Colorectal Dis. 2018;20(3):252-253. doi: 10.1111/codi.13987
10. Web of Science. Sigmoid volvulus. [Accessed November 2018]. Accesible via: http://apps.webofknowledge.com/ Search.do? product $=$ WOS\&SID $=$ D2oaLlaR8ISiJCOwWVi\& search_mode $=$ GeneralSearch\&prID $=\mathrm{d} 224 a e 73-1 \mathrm{e} 82-4 \mathrm{~d} 82$ a085-c664551b3568.

11. Atamanalp SS. Treatment for ileosigmoid knotting: a single-center experience of 74 patients. Tech Coloproctol. 2014;18(3):233-237. doi: 10.1007/s10151-013-1046-3

12. Atamanalp SS. Ileosigmoid knotting: clinical appearance of 73 cases over 45.5 years. ANZ J Surg. 2013;83(1-2):70-73. doi: 10.1111/j.1445-2197.2012.06146.x.

13. Kapadia MR. Volvulus of the small bowel and colon. Clin Colon Rectal Surg 2017;30(1):40-45. doi: 10.1055/s-0036153428.

14. Dolejs SC, Guzman MJ, Fajardo AD, Holcomb BK, Robb BW, Waters JA. Contemporary management of sigmoid volvulus. J Gastrointest Surg. 2018;22(8):1404-1411. doi: $10.1007 /$ s11605-018-3747-4

\section{Authors' Contribution:}

SSA: designed the study, collected and analysed the data, reviewed the literature and prepared the manuscript.

ED: Collected the data, RSA Prepared the manuscript. 